\title{
Moving in waves
}

The nuclei of progenitor cells in the developing neuroepithelium move between the apical and basal surfaces of the cells as they progress through the cell cycle. The functional relevance of this interkinetic nuclear migration (INM) has remained elusive, but Del Bene et al. now show that in the developing retina INM regulates the exposure of neuroepithelial-cell nuclei to Notch, thereby controlling the proportion of cells that exit the cell cycle to undergo differentiation.

The authors set out to delineate the mechanisms that underlie cell-fate choices in the developing retina by studying a zebrafish mutant $\left(m o k^{s 309}\right)$ in which the number of differentiated retinal ganglion cells - the earliest-born cell type in the retina - was increased. Birth dating studies, using sequential injections of thymidine analogues, showed that, compared with wild-type retinal progenitor cells, more $m^{3} k^{s 309}$ mutant cells leave the cell cycle during the first wave of neurogenesis, and thus become retinal ganglion cells. Furthermore, the increased neurogenesis of early-born cell types in $m o k^{s 309}$ mutants was at the expense of later-born cell types, suggesting that mok primarily affects the timing of cell cycle exit and not cell fate directly.

Cloning of the mok gene revealed that it encodes the motor protein dynactin 1. Del Bene et al. wondered whether dynactin 1 is required for INM during eye development and whether abnormal positioning of the progenitor nuclei might underlie the altered retinal cell fate in $m o k^{s 309}$ mutants. Indeed, using live cell imaging, the authors found that in mutant embryos, the nuclei of neuroepithelial cells migrated more basally on average than they did in wild-type zebrafish. However, the cell cycle period of $m o k^{309}$ cells was not altered. Thus, in $m k^{s 309}$ mutants, neuroepithelial-cell nuclei spend a greater proportion of each cell cycle near the basal surface, as a result of which the cells are more likely to be selected for neurogenesis.

How does the abnormal positioning of the nucleus lead to early cell cycle exit? The authors showed that the apical side of the neuroepithelium has higher expression of notch1a, the gene that encodes Notch (which maintains cells in the progenitor state), than the basal side. Accordingly, the activity of a Notch target gene, visualized with a short-lived red fluorescent protein, increased in nuclei moving from the basal to the apical surface and decreased in nuclei moving in the opposite direction, indicating that intracellular Notch signalling also displayed an apical-to-basal gradient.

These data led the authors to propose that dynactin drives INM in neuroepithelial cells and so regulates the level of Notch signalling in the nucleus, which in turn determines whether a cell will exit the cell cycle. With this elegant study they have elucidated the functional significance of INM as a regulator of progenitor cell division and differentiation.

Leonie Welberg

ORIGINAL RESEARCH PAPER Del Bene, F. et al. Regulation of neurogenesis by interkinetic nuclear migration through an apical-basal Notch gradient. Cell 134, 1055-1065 (2008)

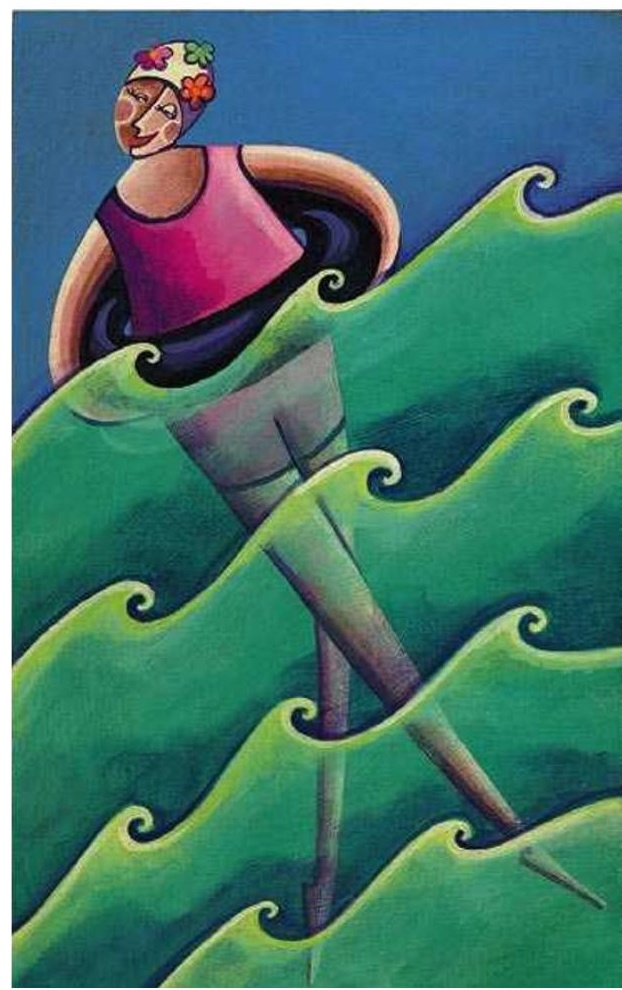

